\title{
Falls among Top Reasons for Transfers from Continuing Care to Acute Care Hospitals in Canada
}

Raymond Przybysz, Heather Dawson and Kira Leeb

\begin{abstract}
There are limited data on the quality and safety of care for residents in continuing care settings. An analysis of the main reasons why residents, 75 and older, of continuing care facilities are transferred to acute care demonstrates that two of the top three reasons for transfers result from potentially avoidable events.
\end{abstract}

n 2004, Baker et al., in their study of adverse events related to hospital care in Canada, found that $7.5 \%$ of those hospitalized in 2000-2001 experienced an adverse event. Since the publication of this study, patient safety within different care settings has been a focus of interest and study. This includes a focus on the safety and quality of care being provided for residents of continuing care settings.

In 2006-2007, there were almost 200,000 Canadians living in homes for the aged, nursing homes, lodges for senior citizens and rest homes in Canada (Statistics Canada 2008). Residents of continuing care facilities are older and more medically complex than ever before (Sharkey 2008; Wagner and Rust 2008). In this article, we concentrate on residents of continuing care settings, aged 75 and older, who were admitted to acute care. We explore patient characteristics and the reasons for admission to hospital for continuing care patients and compare these with factors for seniors admitted to hospital from home or other non-continuing care settings. We note that two of the top three reasons for hospitalizations among residents of continuing care facilities are results of potentially preventable events, and we conclude by highlighting strategies designed to monitor and assess those in continuing care who are at risk for hospitalization for these events.

\section{Methods}

Analyses were based on data for patients 75 years and older obtained from the Canadian Institute for Health Information (CIHI) Discharge Abstract Database for the period of April 1, 2003, to March 31, 2008. Patients were grouped and their characteristics compared based on place of residence upon admission to hospital: continuing care settings versus home or other non-continuing care setting. Continuing care settings included nursing homes, homes for the aged or chronic care facilities (or designated units/beds in the same or another hospital). Ontario's free-standing complex continuing care (CCC) settings or CCC beds in the same or another hospital were also included in this definition.

The most common reasons for hospitalization were determined by major clinical category based on the most responsible diagnosis or the reason that accounted for the majority of the 
hospital stay. Within each major clinical category, patients were further grouped based on their most frequent clinical characteristics. The main reasons for admission and length of stay, including the number of days patients spent designated as alternative level of care (ALC), were compared for those admitted via transfer from a continuing care setting and those admitted from home or other care settings.

\section{Results}

In 2007-2008, 35\% of acute care hospitalizations in Canada (excluding Quebec) were for persons 75 and older; of those, 10\% were for patients transferred from continuing care settings. The top three reasons for these transfers were respiratory conditions (19.8\%) such as pneumonia and chronic obstructive pulmonary disease (COPD); conditions related to the circulatory system $(17.7 \%)$ such as heart failure; and traumatic injury (12.3\%), primarily due to falls. The top three reasons for admissions from home or other settings to acute care differed when compared with those for admissions from continuing care settings. For example, trauma due to falls and other injuries was not among the top three reasons for admission for those admitted from home or other non-continuing care settings (Table 1).

Over a five-year period from 2003-2004 to 2007-2008, there was little change in the percentage of all transfers from continuing care settings attributed to these three reasons (Figure 1), despite increases in the overall number of patient transfers for these reasons (Figure 2).

While length of stay in 2007-2008 did not differ between those transferred from continuing care settings and those admitted from home or from other care settings for injuries due to falls, a larger percentage of patients admitted from home or other non-continuing care settings for fall-related injuries spent time as ALC patients than did those transferred from continuing care settings ( 25.4 versus $15.5 \%$, respectively). In terms of bed-days, patients admitted from continuing care settings for injuries related to falls accounted close to 17,000 bed-days

Table 1. Comparison of reasons for admission*

\begin{tabular}{|c|c|c|}
\hline Diagnosis or Condition & Admitted via Transfer from Continuing Care Setting & Admitted from Home or Other \\
\hline \multirow[t]{5}{*}{ Most common } & Respiratory (19.8\%) & Circulatory $(22.8 \%)$ \\
\hline & Three most frequent clinical characteristics: & Three most frequent clinical characteristics: \\
\hline & Viral/unspecified pneumonia & Heart failure without cardiac catheter \\
\hline & COPD & Arrhythmia without cardiac catheter \\
\hline & Aspiration pneumonia & $\mathrm{Ml} /$ shock/arrest without cardiac catheter \\
\hline \multirow[t]{5}{*}{ Second most common } & Circulatory $(17.7 \%)$ & Digestive (12.7\%) \\
\hline & Three most frequent clinical characteristics: & Three most frequent clinical characteristics: \\
\hline & Heart failure without cardiac catheter & GI hemorrhage \\
\hline & Ml/shock/arrest without cardiac catheter & Symptom/sign of digestive system \\
\hline & Arrhythmia without cardiac catheter & Non-severe enteritis \\
\hline \multirow[t]{5}{*}{ Third most common } & Traumatic injuries $(12.3 \%)$ & Respiratory (12.5\%) \\
\hline & Three most frequent clinical characteristics: & Three most frequent clinical characteristics: \\
\hline & Fixation/repair hip/femur & COPD \\
\hline & Hip replacement with trauma/complication of treatment & Viral/unspecified pneumonia \\
\hline & Fracture of femur & Malignant neoplasm of respiratory system \\
\hline
\end{tabular}

$\mathrm{COPD}=$ chronic obstructive pulmonary disease; $\mathrm{Gl}$ = gastrointestinal; $\mathrm{MI}=$ myocardial infarction .

${ }^{*}$ Based on hospitalizations (not individual patients) for those 75 years and older in Canada, excluding Quebec.

Source: Discharge Abstract Database, 2007-2008, Canadian Institute for Health Information. 
spent as ALC patients, whereas those admitted from home or noncontinuing care settings accounted for just over 86,000 bed-days spent as ALC patients (Table 2).

Again in 2007-2008, one in five patients transferred from continuing care facilities were admitted for respiratory conditions primarily related to pneumonia and COPD in comparison to $12.5 \%$ admitted from home or non-continuing care settings (Table 3 ). Lengths of stay for patients transferred from continuing care facilities and admitted for respiratory conditions also did not differ compared with those admitted from home or noncontinuing care settings.

\section{Discussion}

Continuing care settings in Canada are designed to provide a secure place for individuals requiring 24-hour services such as healthcare, personal care, meals and other housekeeping needs (Health Canada 2004). However, when acute care needs arise, residents need to be transferred to an acute care hospital for assessment and possible admission.

A recent study of the applicability of the concept of potentially avoidable hospitalizations among residents of long-term care facilities suggests that both respiratory conditions such as COPD and pneumonia and injuries due to falls could be considered potentially avoidable hospitalization events for these residents (Walker et al. 2009). In this study, falls and fractures represented $26 \%$ of all hospitalizations considered to be potentially avoidable, whereas COPD and pneumonia combined accounted for $30 \%$. In a report by the Canadian Patient Safety Institute, falls were identified as one of the most frequently reported
Figure 1. Percentage of transfers due to respiratory and circulatory conditions and traumatic injuries*

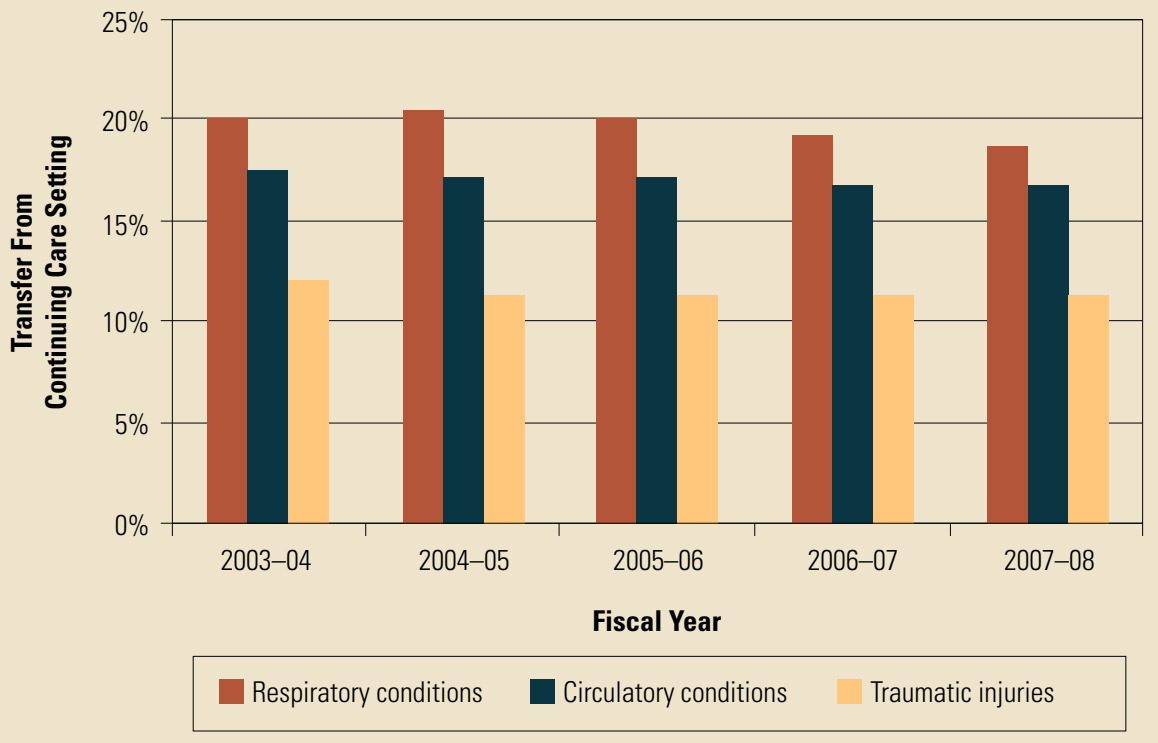

*Based on hospitalizations (not individual patients) of patients 75 years and older in Canada, excluding Quebec.

Source: Discharge Abstract Database, Canadian Institute for Health Information.

Figure 2. Number of transfers due to respiratory and circulatory conditions and traumatic injuries*

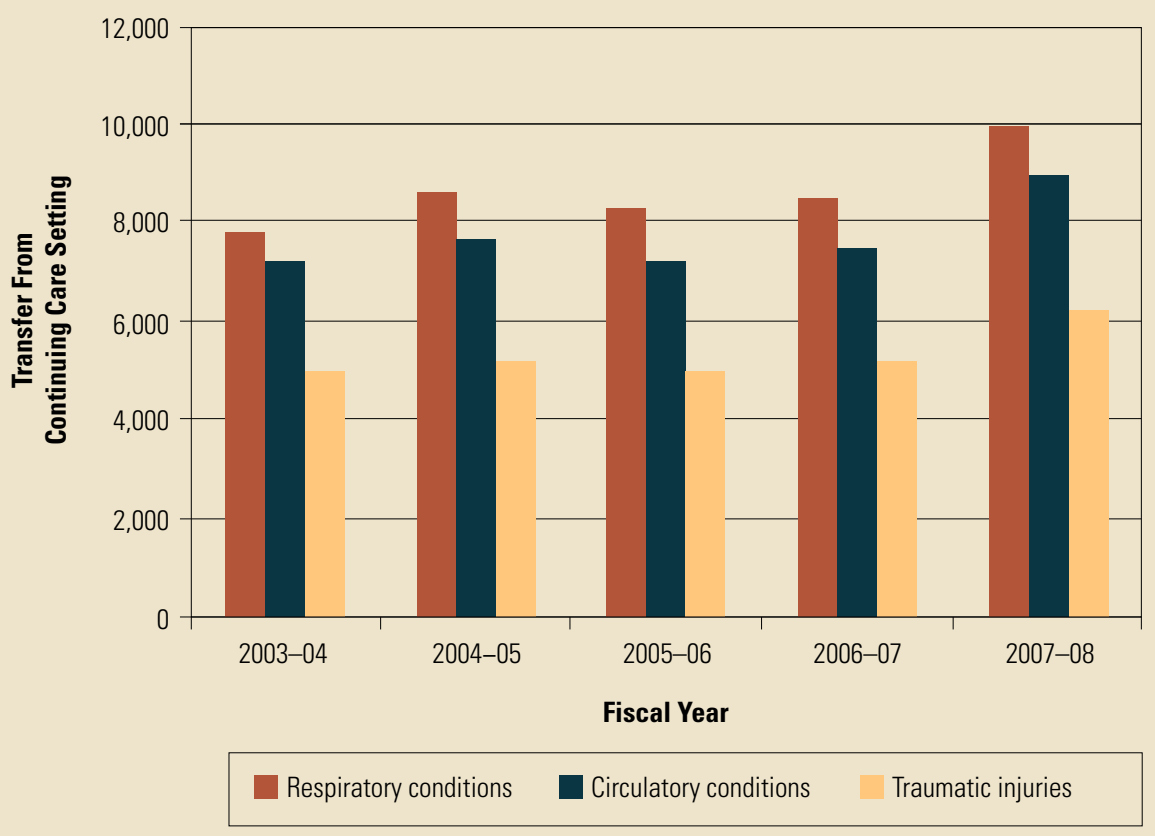

*Based on hospitalizations (not individual patients) of patients 75 years and older in Canada, excluding Quebec

Source: Discharge Abstract Database, Canadian Institute for Health Information. 
Table 2. Hospitalizations for injuries due to falls*

\begin{tabular}{|l|c|c|}
\hline & Admitted via Transfer from Continuing Care & Admitted from Home or Other \\
\hline Total hospitalizations with TI & $6,381(12.3 \%$ of total) & $26,544(6.9 \%$ of total) \\
\hline Hospitalizations due to falls & $5,763(90.3 \%$ of Tl; $11.1 \%$ of total) & 84 \\
\hline Median age (yr) & 87 & 74.2 \\
\hline Percentage female patients & 78.8 & 94.5 \\
\hline Percentage admitted via ED & 95.4 & 8 \\
\hline Median acute LOS $(d)$ & 7 & $4,915(25.4 \%)$ \\
\hline Number with ALC stays ${ }^{\dagger}$ & $856(15.5 \%)$ & $9.2 \%$ of total) \\
\hline Median ALC LOS (d) ${ }^{\dagger}$ & 10.5 & $86,078^{\ddagger}$ \\
\hline Total ALC bed-days ${ }^{\dagger}$ & 16,713 & 9 \\
\hline
\end{tabular}

$A L C=$ alternative level of care; $E D=$ emergency department; $L O S=$ length of stay; $T I=$ traumatic injuries.

* Based on hospitalizations (not individual patients) of patients 75 years and older in Canada, excluding Quebec. Total hospitalizations were 51,730 admitted via transfer from continuing care and 385,762 admitted from home or other non-continuing care setting.

${ }^{\dagger}$ Only among patients identified as ALC (excluding Manitoba).

‡ Excludes two patients who stayed more than a year in ALC.

Source: Discharge Abstract Database, 2007-2008, Canadian Institute for Health Information.

Table 3. Hospitalizations for respiratory conditions*

\begin{tabular}{|l|c|c|}
\hline & $\begin{array}{c}\text { Admitted via Transfer from } \\
\text { Continuing Care Settings }\end{array}$ & Admitted from Home or Other \\
\hline Hospitalizations for respiratory conditions & $10,218(19.8 \%$ of total) & 48,032 (12.5\% of total) \\
\hline Percent admitted via ED & 95.5 & 60.2 \\
\hline Median acute LOS $(d)$ & 6 & $4,027(8.9 \%)$ \\
\hline Number with ALC stays ${ }^{\dagger}$ & $697(7.1 \%)$ & 9 \\
\hline Median ALC LOS $(d))^{\dagger}$ & 8 & 9 \\
\hline
\end{tabular}

$A L C=$ alternative level of care; $E D=$ emergency department; $L O S=$ length of stay.

* Based on hospitalizations (not individual patients) of patients 75 years and older in Canada, excluding Quebec. Total hospitalizations were 51,730 admitted via transfer from continuing care and 385,762 admitted from home or other non-continuing care setting.

${ }^{\dagger}$ Only among patients identified as ALC (excluding Manitoba).

Source: Discharge Abstract Database, 2007-2008, Canadian Institute for Health Information.

adverse events in continuing care and a common reason for seniors' visits to the emergency department (Wagner and Rust 2008). In our analysis, we found that patients admitted from continuing care settings were twice as likely to be admitted due to falls compared with those admitted from home or another setting ( $11.1 \%$ versus $5.2 \%$, respectively).

Knowing who is at risk for falls within continuing care settings may present the most relevant intervention strategy for those caring for residents. Recently, clinical assessment protocols (CAPs) have been developed and released by interRAI to help clinicians assess risk factors among patient populations in continuing care as well as those being provided care in the community (Canadian Institute for Health Information 2008). As falls and injuries related to falls represent a significant health concern, a CAP has been developed to specifically assess the risk for falls. $\mathrm{CIHI}$ is building new CAP reports related to a number of patient 


\section{Patients admitted from continuing care settings were twice as likely to be admitted due to falls compared with those admitted from home or another setting.}

care issues for organizations submitting data to the Continuing Care Reporting System and the Home Care Reporting System. Tracking CAPs triggered over time at individual and organizational levels can inform quality improvement initiatives and shed light on the effectiveness of the care provided. HQ

\section{References}

Baker, G.R., P.G. Norton, V. Flintoft, R. Blais, A. Brown, J. Cox, E. Etchells, W.A. Ghali, P. Hébert, S.R. Majumdar, M. O’Beirne, L. Palacios-Derflingher, R.J. Reid, S. Sheps and R. Tamblyn. 2004. "The Canadian Adverse Events Study: The Incidence of Adverse Events among Hospital Patients in Canada." Canadian Medical Association Journal 170(11): 1678-86.

Canadian Institute for Health Information. 2008. InterRAI Clinical Assessment Protocols (CAPs). Ottawa, ON: Author. Retrieved January 12, 2008. <http://secure.cihi.ca/cihiweb/dispPage.jsp?cw_ page=RC_2109_E $>$.

Health Canada. 2004. What Is Long-Term Facilities-Based Care? Ottawa, ON: Author. Retrieved November 26, 2008. <http://www. hc-sc.gc.ca/hcs-sss/home-domicile/longdur/index-eng.php $>$.

Sharkey, S. 2008. People Caring for People: Impacting the Quality of Life and Care of Residents of Long-Term Care Homes - A Report of the Independent Review of Staffing and Care Standards for Long-Term Care Homes in Ontario. Toronto, ON: Ministry of Health and Long-Term Care. Retrieved November 18, 2008. <http:/www.health.gov.on.ca/ english/public/pub/ministry_reports/staff_care_standards/staff_care_ standards.pdf>.
Statistics Canada. 2008. Residential Care Facilities 2006-2007 (Catalogue No. 83-237-X). Ottawa, ON: Author.

Wagner, L.M. and T.B. Rust. 2008. Safety in Long-Term Care Settings. Edmonton, AB: Canadian Patient Safety Institute.

Walker, J.D., G.F. Teare, D.B. Hogan, S. Lewis and C.J. Maxwell. 2009. "Identifying Potentially Avoidable Hospital Admissions from Canadian Long-Term Care Facilities.” Medical Care 47(2): 1-5.

\section{About the Authors \\ Raymond Przybysz, MSc, is project lead of health services research at Canadian Institute for Health Information $(\mathrm{CIHI})$. He is responsible for coordination and implementation of project plans and provides support in the field of analytical methods and research techniques. He has previously worked with several research institutes in Ontario and Quebec.}

Heather Dawson, MHSc, is manager of health services research at $\mathrm{CIHI}$. In this role, Heather oversees the development of health services analyses with the view to addressing relevant questions for policy and health systems issues. She has previously worked in leadership roles in hospitals and health planning and advocacy organizations in Ontario.

Kira Leeb is director of health system analysis at CIHI. Kira has been with $\mathrm{ClHI}$ since 2000 and has been involved in the production of many reports about various aspects of Canada's healthcare system over the years.

Words from this article presented in order of popularity.

accounted acute admission admitted adverse alc assess aviodable based canada canadian cihi clinical compared conditions continuing adaines ate events facilites falls health home hospital motemiter injuries imerute

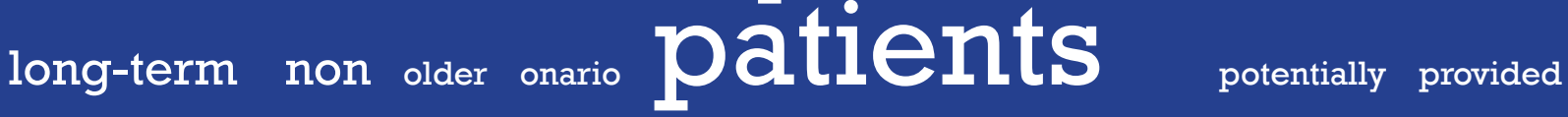
reasons related reports residents respiratory risk safety servicest Settings spent study top transferred 